\title{
Relative validation of a food frequency questionnaire against diet records to estimate $n-3$ fatty acid intake in adults
}

\author{
J. McKenzie, L. Muirhead, E. Shillinglaw and I. Paciarotti \\ Queen Margaret University, Edinburgh EH21 6UU, UK
}

Long chain omega-3 PUFA (LC $n$-3 PUFA) are important for health and well-being. Recent studies suggest in vivo synthesis of LC $n$-3 PUFA from their dietary precursor, $\alpha$-linolenic acid (ALA), may be limited. Preformed LC $n-3$ PUFA are found in a limited number of foods that are not commonly eaten in the UK diet. The potential for insufficient conversion of ALA, which is found in plentiful supply in the diet, has therefore raised questions as to whether intakes of LC $n-3$ PUFA are sufficient. Very little information is currently available on intakes of LC $n-3$ PUFA and, consequently, there is a need to develop an efficient method to estimate intakes. As intakes of LC $n-3$ PUFA in the diet are somewhat sporadic, an accurate assessment of intake would traditionally involve recording dietary intake over a long period of time. This approach is not efficient for use in many population groups. An FFQ has previously been developed to assess children's total $n$-3 PUFA intake ${ }^{(1,2)}$, including intakes of DHA and EPA, and estimated intakes appear to be comparable to intakes reported in the National Diet and Nutrition Survey ${ }^{(3)}$.

The aim of this study was to investigate the relative validity of the FFQ in comparison to a 14-d diet record in estimating $n-3$ PUFA intakes in adults aged 19 to 64 years.

Forty-four participants ( 24 females) completed a 14-d diet record, followed by the FFQ. Dietary intakes were estimated manually using nutrient composition tables and modification of the FFQ spreadsheet to account for adult portion sizes. Comparison of methods by BlandAltman analysis indicated that the FFQ over estimated total $n-3$ PUFA intakes by an average of $0.6 \mathrm{~g} / \mathrm{d}$, yet underestimated intakes of LC $n$-3 PUFA by an average of $30 \mathrm{mg} / \mathrm{d}$. Agreement between the methods was very good for both total $n$ - 3 PUFA intake and total LC $n-3$ PUFA intake. Only two subjects consistently showed discrepancies outside the $95 \%$ limits of agreement, due to exceptionally high intakes being reported. Results were compared with the SACN recommendations ${ }^{(4)}$ indicating that, when assessed by either method, $82 \%$ of subjects had intakes below the $450 \mathrm{mg} / \mathrm{d}$ recommendation.

The results of the study showed the FFQ to be a good tool to establish LC $n$-3 PUFA intakes in groups of adults; however, its use in assessing the adequacy of individual intakes may be limited. Further validation, potentially against a biomarker of fatty acid intake, is warranted.

1. McGregor A (2008) Design of a food frequency questionnaire to measure $n$-3 fatty acids intake in children. Master Thesis, Queen Margaret University.

2. Paciarotti I (2009) Re-evaluation of an existing food frequency questionnaire to reflect total $n-3$ fatty acids intake in primary school children. Honours Project, Queen Margaret University.

3. Gregory JR, Lowe S, Bates CJ et al. (2000) National Diet and Nutrition Survey: Young People Aged 4-18 Y ears. Volume 1: Report of the Diet and Nutrition Survey. London: TSO.

4. Scientific Advisory Committee on Nutrition (SACN). 2004. Advice on Fish Consumption: Benefits and Risks. London: TSO. 